\title{
AVALIAÇÃO DA HETEROGENEIDADE DO SINTER FEED DA ARCELORMITTAL MINA DE SERRA AZUL - VIA VARIOGRAMA*
}

Juliana Cecília Costa Ribeiro Vieira' Luana Gonçalves Guimarães² Luiz Paulo Serrano

\section{Resumo}

As operações de amostragem de qualquer atividade que necessite de controle de qualidade são fundamentais tanto para fins de garantia da qualidade quanto para efeito comercial dos produtos. Na mineração de ferro não é diferente. Uma amostragem que carregue um vício de $0,20 \%$ no teor de Fe (\%) pode representar uma perda de faturamento de aproximadamente US\$226 mil para cada milhão de toneladas comercializadas aos preços atuais. Para fazer um bom plano de amostragem é necessário conhecer o grau de heterogeneidade ou variação de qualidade $(\sigma \mathrm{w})$ do minério a ser amostrado. Segundo Pierre Maurice Gy, um dos mais renomados especialistas em amostragem de minérios em geral, a forma mais indicada para estimar a $\sigma \mathrm{w}$ é o método do variograma. Neste trabalho usamos a metodologia do variograma, com base na Norma Técnica ABNT ISO 3084:2003, para estimar a $\sigma \mathrm{w}$ do minério tipo Sinter Feed produzido na Mina de Serra Azul da ArcelorMittal.

Palavras-chave: Mineração; Variação de Qualidade; Variograma, Heterogeneidade.

\section{HETEROGENEITY ESTIMATION OF IRON ORE TYPE SINTER FEED OF ARCELORMITTAL (SERRA AZUL MINE ) BY THE VARIOGRAM METHOD}

\section{Abstract}

The operations of sampling of any activity that requires quality control are fundamental both for quality assurance purposes and for the commercial effect of the products. In iron mining it is no different. A sample that carries a defect of $0.20 \%$ in Fe content (\%) may represent a loss of revenue of approximately US $\$ 226,000$ for each million tons traded at current prices. To make a good sampling plan it is necessary to know the degree of heterogeneity or quality variation ( $\sigma \mathrm{w}$ ) of the ore to be sampled. According to Pierre Maurice Gy, one of the most renowned specialists in ore sampling in general, the best way to estimate $\sigma w$ is the variogram method. In this work we use the variogram methodology, based on the Technical Standard ABNT ISO 3084:2003, to estimate the $\sigma \mathrm{w}$ of the Sinter Feed type ore produced at ArcelorMittal's Serra Azul Mine.

Keywords: Mining; Quality Variation; Variogram, Heterogeneity.

1 Graduada em Ciência Farmacêuticas e Técnica em química Industrial, supervisora de laboratório na ArcelorMittal Mineração Serra Azul, membro da Comissão de Estudos de Amostragem do CB41 Comitê Brasileiro de Minério de Ferro da ABNT, Itatiaiuçu, Minas Gerais, Brasil.

2 Graduada em Gestão da Produção industrial e técnica em mineração, Técnica de beneficiamento na ArcelorMittal Mineração Serra Azul, Itatiaiuçu, Minas Gerias, Brasil.

3 Engenheiro químico, Bacharel em estatística, membro da Comissão de Estudos de Amostragem do CB41 Comitê Brasileiro de Minério de Ferro da ABNT e Consultor de métodos estatísticos para mineração, Belo Horizonte, Minas Gerais, Brasil.. 


\section{INTRODUÇÃO}

A mineração é um dos setores básicos da economia do país, contribui de forma decisiva para o bem estar e a melhoria da qualidade de vida das pessoas, além de fundamental para o desenvolvimento de uma sociedade igualitária, desde que operada com responsabilidade social e sempre presentes os preceitos do desenvolvimento sustentável.

Nesse contexto, a ArcelorMittal Mineração Brasil, desempenha suas atividades, atualmente, a partir de duas minerações de Minério de Ferro: Mina de Serra Azul, situada em Itatiaiuçu-MG, e Mina do Andrade, em Bela Vista de Minas $M G$, sendo a primeira, o local de nosso objeto de estudo.

A Mina de Serra Azul, localizada no município de Itatiaiuçu, tem capacidade instalada de 680 mil toneladas/ano de minério de ferro granulado, destinadas ao mercado interno e às empresas do grupo ArcelorMittal no Brasil, e de 3,5 milhões de toneladas de minério de ferro fino (sinter feed), destinadas a empresas do Grupo ArcelorMittal no exterior. As operações da empresa iniciaram-se em 1974, sendo que em 2008 foi comprada pela ArcelorMittal, juntando-se ao Grupo. Possui significativo potencial geológico, com uma área licenciada de 620 hectares e base de recursos de 1,2 bilhões de toneladas de ROM (minério bruto). A mina produz o minério de ferro sinter feed (SF), que atende à demanda do mercado interno e também do mercado externo, e o lump ore, atendendo à demanda das empresas do grupo.

Havia um problema com um dos consumidores do nosso Sinter Feed quanto à diferença, não aceitável, dos resultados de Ferro total entre um lote que era embarcado em Sarzedo (terminal de carregamento dos produtos da ArcelorMittal Serra Azul) e o seu recebimento no destino final, com um agravante que os resultados que compunham um navio para exportação carregavam um vício[6] de $0,40 \%$ em $\mathrm{Fe}$ total, ou seja, os resultados do cliente tinham uma tendência sistemática de apresentarem resultados menores do que os nossos, gerando perda de faturamento e multas por problemas de qualidade.

A discussão era de forma difícil porque não havia um parâmetro que definisse qual a diferença entre os resultados de Fe total no carregamento e na descarga, que pudesse ser considerada como estatisticamente correta ou se realmente havia erro de amostragem. Esse parâmetro é a precisão da amostragem ou intervalo de confiança para estimar o valor real da característica de qualidade de interesse.

A metodologia de amostragem em mineração tem três características fundamentais que a torna diferente das demais amostragens, são:

(a) O grau de heterogeneidade do material a ser amostrado, é difícil de ser medido, uma vez que analisar todas as unidades amostrais (incrementos) individualmente é operacionalmente impossível no dia a dia;

(b) A delimitação da massa da unidade amostral (incremento) não é uma tarefa trivial. É necessário conhecimento prévio da natureza geológica do material e da precisão (margem de erro) relativa à característica de qualidade de interesse;

(c) Uma vez coletados os incrementos necessários a uma determinada margem de erro estabelecida no plano de amostragem, esses incrementos são homogeneizados, dentro de premissas bem definidas por normas técnicas ${ }^{[3]}$, e uma nova amostragem é realizada, quando se extrai a amostra final para análise. No caso da característica de qualidade teor de Ferro total, a massa a ser efetivamente analisada é de apenas $1 \mathrm{~g}$, ou seja, para um lote de 10.000 toneladas a massa 
efetivamente analisada é de apenas $1 \mathrm{~g}$ o que guarda uma relação de 1 para 10.000.000.000 possibilidades de se extrair essa pequena massa.

A importância fundamental da determinação de $\hat{\sigma}_{W}$ fica demonstrada pela teoria básica de amostragem. Seguindo a orientação de Pierre Gy ${ }^{[1,2]}$, deve ser determinada pelo método do variograma.

\subsection{Teoria básica de amostragem ${ }^{[7,8]}$}

Intervalo de Confiança para a Média Real é dado pela equação 1.

onde:

$$
\boldsymbol{\mu}=\bar{X} \pm Z_{\alpha} \widehat{\boldsymbol{\sigma}}_{\bar{X}}
$$

$\mu$ é o valor real a ser estimado da característica de qualidade de interesse;

$\bar{X}$ é o resultado obtido pelo laboratório de $n$ incrementos coletados;

$Z_{\alpha}$ é uma variável padrão que determina o nível de confiança a ser adotado para a amostragem;

$\hat{\sigma}_{\bar{X}}$ é a variabilidade dos valores médios da característica de qualidade de interesse;

$n$ é o numero de incrementos.

Significado prático de $\boldsymbol{Z}_{\boldsymbol{\alpha}}$ :

Segundo a norma técnica ABNT NBR ISO 3082 [3] um nível de confiança de $95 \%$ é suficiente. Neste caso o valor de $Z_{\alpha}=1,96 \cong 2$, o que significa que a cada 100 amostragens 5 (cinco) podem sair fora dos limites projetados mesmo que os todos os procedimentos de amostragem, preparação e análise das amostras tenham sido bem executados.

Pelo Teorema do Limite Central - TLC ${ }^{[7,8]}$ temos as equações 2 e 3:

$$
\begin{array}{r}
\widehat{\sigma}_{\bar{X}}=\frac{\widehat{\sigma}_{W}}{\sqrt{n}} \\
\mu=\bar{X} \pm Z_{\alpha} \frac{\widehat{\sigma}_{W}}{\sqrt{n}}
\end{array}
$$

Onde: $\hat{\sigma}_{W}$ é a estimativa do grau de heterogeneidade da característica da qualidade de interesse, ou seja, quanto menor for $\hat{\sigma}_{W}$ menos heterogêneo é o produto (mais homogêneo).

\section{Precisão de Amostragem $\left(\boldsymbol{\beta}_{\boldsymbol{S}}\right)$ :}

é o intervalo que se estima para a diferença entre o valor de $\mu$ e $\bar{X}$, é dado pela equação 4, 5 e 6

$$
\begin{array}{cr} 
& \boldsymbol{\beta}_{S}=|\boldsymbol{\mu}-\overline{\boldsymbol{X}}| \\
\text { Então: } & |\boldsymbol{\mu}-\overline{\boldsymbol{X}}|=\boldsymbol{Z}_{\alpha} \frac{\widehat{\sigma}_{W}}{\sqrt{n}} \\
\text { Logo: } & \boldsymbol{\beta}_{S}=\boldsymbol{Z}_{\alpha} \frac{\widehat{\sigma}_{W}}{\sqrt{n}}
\end{array}
$$




\section{MATERIAIS E MÉTODOS}

\subsection{Planejamento}

O experimento foi conduzido pelos setores de Laboratório Industrial e Usina de Concentração de Minério de Ferro da Mina de Serra Azul. As amostras foram coletadas, utilizando um amostrador tipo Cross -Belt, confeccionado e utilizado de acordo com a norma ABNT NBR 16595:2017.

A partir da produção da planta de beneficiamento foram tomados incrementos de duas em duas horas, ou seja, a cada intervalo a produção foi de aproximadamente 300 toneladas de SF, totalizando uma produção final de aproximadamente 11.000 toneladas. As 36 amostras assim coletadas foram acondicionadas em sacos plásticos identificados, lacradas e encaminhadas ao laboratório para preparação e análise química.

No laboratório as amostras foram cadastradas e receberam um código de rastreabilidade, foram pesadas individualmente e todas as amostras foram secas em estufa a $105^{\circ} \mathrm{C} \pm 5^{\circ} \mathrm{C}$, massa seca foi utilizada para determinação da umidade. A massa de cada incremento foi em média de $3,5 \mathrm{~kg}$ com Coeficiente de Variação $(\mathrm{CV})^{[3]}$ de $12 \%$ atendendo a especificação das normas ISO $^{[3]}$, conforme tabela 01.

Após secagem, o material foi homogeneizado e quarteado, em quarteador tipo carrossel, uma parte do material foi separada para analise granulometrica e a outra para prosseguir na preparação para analises químicas. A analise granulométrica foi realizada conforme Norma ISO ABNT 4701:2009. O material destinado a analise química,foi pulverizado em pulverizador de Panela, tipo LM2 da ESA, com capacidade para $1200 \mathrm{~g}$ de material, após foi quarteado e novamente pulverizado em pulverizado de panela, capacidade de $100 \mathrm{cc}$, da Dialmática. O material pulverizado, cerca de 50 gramas, foi colocado em pacotes identificados e encaminhados ao laboratório químico.

As amostras foram analisadas de forma aleatória, conforme tabela 02 , no menor espaço de tempo possível, de forma a evitar as incertezas inerentes do processo. Para este trabalho iremos considerar somente as analises do elemento Ferro.

As analises químicas foram feitas por fluorescência de $\mathrm{Rx}$, utilizando fusão para confecção das pastilhas. Na preparação da pastilha foram utilizados 0,6 gramas de amostra para 6,0 gramas de Tetraborato de sódio. A leitura foi feita no equipamento Axios Minerals da Panalytical. A curva de calibração do equipamento é constituída por padrões internacionais e blends destes, contendo ao todo 170 padrões, sem aplicação de qualquer tipo de correção, com exceção da correção alfa de Sódio ( $\mathrm{Na}$ ) para todos os elementos. O controle da aplicação é feito diáriamente utilizando padrões que não fazem parte da constituição da curva.

Os 36 resultados, assim obtidos, foram submetidos ao método do variograma simplificado e geral, conforme norma ABNT ISO 3084:2003, e utilizado o maior valor obtido para representar o grau de heterogeneidade $\left(\sigma_{w}\right)$ do minério tipo Sinter Feed.

Reforçando que sem a estimativa de $\sigma_{w}$ não é possível fazer um plano de amostragem para qualquer tipo de minério. 
Tabela 1: Massa individual de cada incremento

\begin{tabular}{|c|c|c|c|}
\hline $\begin{array}{c}\mathbf{N}^{\circ} \text { da } \\
\text { amostra }\end{array}$ & Peso (kg) & $\begin{array}{c}N^{\circ} \text { da } \\
\text { amostra }\end{array}$ & Peso (kg) \\
\hline 1 & 3,92 & 19 & 2,40 \\
\hline 2 & 4,93 & 20 & 4,00 \\
\hline 3 & 5,43 & 21 & 4,20 \\
\hline 4 & 4,23 & 22 & 3,20 \\
\hline 5 & 3,07 & 23 & 4,65 \\
\hline 6 & 4,10 & 24 & 3,20 \\
\hline 7 & 4,92 & 25 & 3,35 \\
\hline 8 & 4,30 & 26 & 4,00 \\
\hline 9 & 3,50 & 27 & 4,42 \\
\hline 10 & 4,60 & 28 & 4,37 \\
\hline 11 & 4,00 & 29 & 4,13 \\
\hline 12 & 3,75 & 30 & 4,28 \\
\hline 13 & 3,95 & 31 & 5,70 \\
\hline 14 & 4,10 & 32 & 4,00 \\
\hline 15 & 5,00 & 33 & 3,15 \\
\hline 16 & 3,40 & 34 & 3,50 \\
\hline 17 & 4,48 & 35 & 3,53 \\
\hline 18 & 4,32 & 36 & 3,33 \\
\hline
\end{tabular}

Tabela 2: Aleatorização das amostras para realização das análises

\begin{tabular}{|c|c|c|c|}
\hline $\begin{array}{c}\text { Ordem de } \\
\text { analise }\end{array}$ & $\begin{array}{l}\mathbf{N}^{\circ} \text { da } \\
\text { amostra }\end{array}$ & $\begin{array}{c}\text { Ordem de } \\
\text { analise }\end{array}$ & $\begin{array}{c}\mathrm{N}^{\circ} \text { da } \\
\text { amostra }\end{array}$ \\
\hline 1 & 13 & 19 & 25 \\
\hline 2 & 32 & 20 & 33 \\
\hline 3 & 3 & 21 & 30 \\
\hline 4 & 7 & 22 & 23 \\
\hline 5 & 29 & 23 & 35 \\
\hline 6 & 12 & 24 & 27 \\
\hline 7 & 36 & 25 & 24 \\
\hline 8 & 9 & 26 & 6 \\
\hline 9 & 16 & 27 & 19 \\
\hline 10 & 34 & 28 & 21 \\
\hline 11 & 17 & 29 & 8 \\
\hline 12 & 2 & 30 & 20 \\
\hline 13 & 14 & 31 & 18 \\
\hline 14 & 5 & 32 & 28 \\
\hline 15 & 10 & 33 & 11 \\
\hline 16 & 15 & 34 & 31 \\
\hline 17 & 26 & 35 & 1 \\
\hline 18 & 4 & 36 & 22 \\
\hline
\end{tabular}




\subsection{Determinação do Variograma Experimental $\left(V_{E}(t){ }^{[4]}\right.$}

O valor do variograma experimental em cada passo $\left(V_{E}\right)$ é dado pela equação 8.

t é dado por $k \Delta t$ ( $\Delta t$ é o intervalo de amostragem);

$$
V_{E}(t)=\frac{\sum_{i=1}^{N_{k}}\left(\bar{x}_{i+k}-\bar{x}_{i}\right)^{2}}{2 N_{k}}
$$

$N_{\mathrm{k}}$ é o número de pares de incrementos, n-k no passo k;

$X_{i+k}$ é o resultado da análise para o incremento i+k;

$X_{i}$ é o resultado da análise do incremento i.

\subsection{Determinação do Variograma Corrigido $\left(V_{C}(t)\right)^{[4]}$}

Para eliminar os erros de preparação e análise deve-se subtrair de cada passo do variograma experimental $\left(\mathrm{V}_{\mathrm{E}}\right)$ o valor de $\frac{\widehat{\sigma}_{P M}^{2}}{2}$, que deve ser obtido de acordo com a

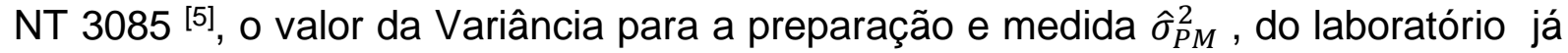
foi determinado, assim temos o Variograma corrigido, conforme equação 9.

$$
V_{c}(t)=V_{E}(t)-\frac{\widehat{\sigma}_{P M}^{2}}{2}
$$

\subsection{Determinação da Variação de qualidade}

A variação de qualidade é determinada pelas fórmulas abaixo (todos os cálculos foram realizados no ambiente $R{ }^{[9,10]}$ ):

Na prática os variogramas podem ser ajustados por uma linha reta, é definido pela equação 10.

$$
V_{C}(t)=V_{0}+B t
$$

onde:

$V_{0}$ é o componente aleatório da variância do variograma (corresponde ao intercepto da linha reta experimental) e $B$ é a inclinação (ou gradiente) do variograma (corresponde ao coeficiente angular da linha reta experimental).

$V_{0}$ é determinado segundo a equação 11 .

$$
V_{0}=\bar{V} c(t)+B * \bar{p}
$$

Onde $\bar{V} c(t)$ e $\bar{p}$ são respectivamente as médias para os variogramas corrigidos e a média do número de passos e $B$ é determinado segundo a equação 12 .

$$
B=\frac{\sum_{i=1}^{n} p_{i} * V_{c i}-n * \bar{p} * \bar{V} c(t)}{\sum_{i=1}^{n} p_{i}^{2}-n * \bar{p}^{2}}
$$

A variância estimada da amostragem $\left(\hat{\sigma}_{s}^{2}\right)$ para amostragens sistemáticas é dada pela equação 13 onde $\mathrm{T}$ é a tonelagem total do lote.

$$
\hat{\sigma}_{S}^{2}=\frac{V_{0}}{n}+\frac{B T}{6 n^{2}}
$$

Substituindo: $\frac{T}{n}$ por $\Delta t$, ou seja, intervalo entre as amostragens temos a equação 14: 


$$
\hat{\sigma}_{s}^{2}=\frac{V_{0}}{n}+\frac{B \Delta t}{6 n}
$$

A relação entre o variograma e a estimativa de variação de qualidade $\left(\hat{\sigma}_{w}\right)$ é dada pelas equações 15 e 16.

$$
\begin{aligned}
& \hat{\sigma}_{S}^{2}=\frac{\widehat{\sigma}_{w}^{2}}{n} \\
& \text { Logo: } \quad \hat{\sigma}_{w}=\sqrt{V_{0}+\frac{B \Delta t}{6}} \rightarrow \text { passo a passo } \Delta t=1 \rightarrow \hat{\sigma}_{w}=\sqrt{V_{0}+\frac{B}{6}}
\end{aligned}
$$

\subsection{Determinação da Precisão Global de Amostragem}

A determinação da Precisão de Amostragem $\left(\beta_{\mathrm{s}}\right)$ do Sinter Feed ${ }^{[5]}$ produzido na planta de beneficiamento, com nível de confiança de $95 \%$, conforme recomendação das normas NBR ISO de amostragem é dado pela equação 6.

A precisão de preparação e análise $\left(\beta_{P M}\right)$ foi determinada pelo laboratório da

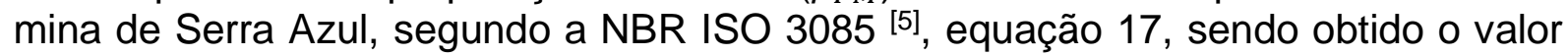
de $0,38 \%$.

$$
\beta_{P M}=Z_{\alpha} * \sigma_{P M} \rightarrow Z_{\alpha} * \sqrt{\frac{1}{2 n_{2}} \sum R_{P M}^{2}}
$$

Sendo: $n_{2}$ : número de pares de amostras tomadas para realização da estimativa de precisão de preparação e análise (laboratório);

$R_{P M}^{2}$ : quadrado da amplitude entre um par de amostras teste;

$\sigma_{P M}$ : desvio padrão dos pares de amostras testes;

$z_{\alpha}$ : variável padrão que define o nível de confiança para $\beta_{P M}$. 18.

Logo, a precisão global do sistema de amostragem é de definida pela equação

$$
\beta_{S P M}=\sqrt{\beta_{S}^{2}+\beta_{P M}^{2}}
$$

\section{RESULTADOS E DISCUSSÃO}

\subsection{Determinação do Variograma}

\begin{tabular}{|c|c|c|c|c|c|c|c|c|c|}
\hline № & $\% \mathrm{Fe}$ & № & $\% \mathrm{Fe}$ & № & $\% \mathrm{Fe}$ & № & $\% \mathrm{Fe}$ & № & $\% \mathrm{Fe}$ \\
\hline 1 & 63,77 & 9 & 60,57 & 17 & 63,01 & 25 & 62,21 & 33 & 60,47 \\
\hline 2 & 63,17 & 10 & 61,11 & 18 & 63,37 & 26 & 61,79 & 34 & 62,14 \\
\hline 3 & 64,11 & 11 & 60,74 & 19 & 62,89 & 27 & 61,53 & 35 & 61,67 \\
\hline 4 & 63,53 & 12 & 63,15 & 20 & 61,52 & 28 & 63,1 & 36 & 62,63 \\
\hline 5 & 63,73 & 13 & 62,43 & 21 & 62,7 & 29 & 62,47 & & \\
\hline 6 & 63,65 & 14 & 61,89 & 22 & 62,78 & 30 & 62,54 & & \\
\hline 7 & 63,31 & 15 & 62,02 & 23 & 63,1 & 31 & 62,36 & & \\
\hline 8 & 62,62 & 16 & 61,93 & 24 & 62,14 & 32 & 62,39 & & \\
\hline
\end{tabular}

A tabela 3, apresenta os resultados das analises de ferro, para cada uma das 36 amostras coletadas.

Tabela 3: Resultado das Análises de Ferro 
Para o laboratório da mina o valor determinado para $\frac{\widehat{\sigma}_{P M}^{2}}{2}$ é de $\mathbf{0 , 0 1 8 1}$.

Passo 1: $V_{E}=\left[(63,17-63,77)^{2}+(64,11-63,17)^{2}+(63,53-64,11)^{2}+\ldots+(62,63-61,67)^{2}\right] /$ $(2 \times 35)=0,4298$

$V_{C}=0,4298-0,0181=\mathbf{0 , 4 1 1 7} \rightarrow$ Passo 1

Passo 2: $V_{E}=\left[(64,11-63,77)^{2}+(63,53-63,17)^{2}+(63,73-64,11)^{2}+\ldots+(62,63-\right.$

$\left.62,14)^{2}\right] /(2 \times 34)=0,5802$

$V_{C}(\Delta t) 2=0,5802-0,0181=\mathbf{0 , 5 6 2 1} \rightarrow$ Passo 2

E assim sucessivamente até completar os 35 passos possíveis do variograma com 36 amostras, tabela 4 apresenta o resultado de todos os passos do variograma corrigido, a figura 01 representa o gráfico do variograma geral.

Tabela 4: Resultados passo a passo do variograma corrigido (Vc)

\begin{tabular}{|c|c|c|c|}
\hline Passos & $\begin{array}{l}\text { Variograma } \\
\text { Corrigido }\left(\mathrm{V}_{c}\right)\end{array}$ & Passos & $\begin{array}{l}\text { Variograma } \\
\text { Corrigido }\left(\mathrm{V}_{\mathrm{c}}\right)\end{array}$ \\
\hline 1 & 0,412 & 19 & 10,471 \\
\hline 2 & 0,562 & 20 & 10,151 \\
\hline 3 & 0,835 & 21 & 12,194 \\
\hline 4 & 0,814 & 22 & 0,958 \\
\hline 5 & 0,81 & 23 & 11,222 \\
\hline 6 & 0,839 & 24 & 0,942 \\
\hline 7 & 0,987 & 25 & 13,131 \\
\hline 8 & 12,313 & 26 & 15,99 \\
\hline 9 & 0,951 & 27 & 18,045 \\
\hline 10 & 0,925 & 28 & 21,295 \\
\hline 11 & 0,677 & 29 & 22,803 \\
\hline 12 & 0,899 & 30 & 27,065 \\
\hline 13 & 0,813 & 31 & 26,459 \\
\hline 14 & 0,909 & 32 & 33,317 \\
\hline 15 & 0,809 & 33 & 22,765 \\
\hline 16 & 0,712 & 34 & 26,341 \\
\hline 17 & 0,796 & 35 & 21,814 \\
\hline 18 & 0,823 & & \\
\hline
\end{tabular}

\subsection{Resultado da Variação de qualidade}

Os resultados para a Variação de qualidade bem com as expressões utilizadas estão na tabela 5 . 
Tabela 5: Resultados Obtidos

\begin{tabular}{c|c|c}
\hline & Resultados & Equações \\
\hline $\boldsymbol{V}_{\boldsymbol{0}}$ & 0,7489 & 11 \\
\hline $\mathbf{B}$ & 0,0077 & 12 \\
\hline$\hat{\sigma}_{w}$ & $0,87 \%$ & 16 \\
\hline
\end{tabular}

\subsection{Resultado da Precisão Global de Amostragem}

Os resultados para determinação da precisão global estão na tabela 6 .

Tabela 6: Resultados Obtidos

\begin{tabular}{c|c|c}
\hline & Resultados & Equações \\
\hline$\beta_{\mathrm{S}}$ & $0,35 \%$ & 6 \\
\hline$\beta_{\mathrm{PM}}$ & $0,38 \%$ & 17 \\
\hline$\beta_{\mathrm{SPM}}$ & $0,52 \%$ & 18 \\
\hline
\end{tabular}

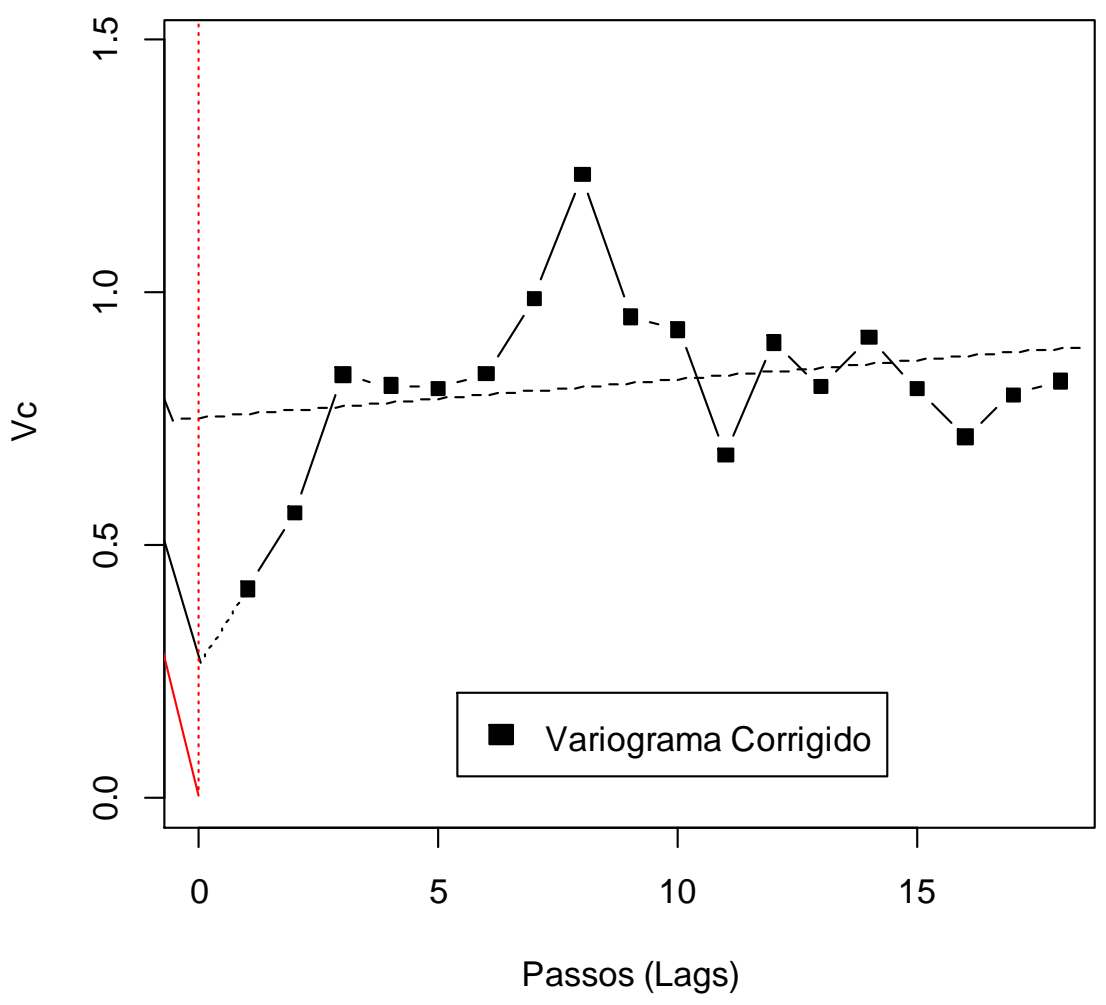

Figura 1 - Método Variograma Geral 


\section{CONCLUSÃo}

$\mathrm{Na}$ pesquisa bibliográfica que pesquisamos em nível de Brasil não encontramos nenhum trabalho que avalie o grau de heterogeneidade de produtos de minério de ferro tipo Sinter Feed via variograma.

O produto Sinter Feed da ArcelorMittal Serra Azul apresenta um baixo grau de heterogeneidade, conforme podemos observar na tabela 7 , o que reflete todo os esforços do controle de qualidade da Mina e da Usina quanto a um produto que atenda as especificações dos clientes e está dentro das exigências das normas internacionais que regem o comércio de minério de ferro no exterior.

Tabela 7: Comparativo entre NBR ISO ${ }^{[3]}$ e ArcelorMittal Serra Azul

\begin{tabular}{|c|c|c|c|c|c|}
\hline \multirow{2}{*}{$\begin{array}{c}(\%) \\
\text { Teor Ferro }\end{array}$} & \multicolumn{2}{|c|}{ Classificação de Variação de Qualidade } & \multicolumn{2}{c|}{ Precisões } \\
\cline { 2 - 6 } & Grande & Média & Pequena & $\beta_{S}$ & $\boldsymbol{\beta}_{S P M}$ \\
\hline \multirow{2}{*}{ NBR ISO } & $\hat{\sigma}_{w}>2,0$ & $\begin{array}{c}2,0>\hat{\sigma}_{w} \\
\geq 1,5\end{array}$ & $\hat{\sigma}_{w}<1,5$ & 0,50 & 0,55 \\
\hline ArcelorMittal & \multicolumn{2}{|c|}{ Pequena } & $\hat{\sigma}_{w}=0,87$ & 0,35 & 0,52 \\
\hline
\end{tabular}

Temos agora todos os elementos necessários para apresentar e discutir com qualquer dos nossos clientes quanto à certificação da qualidade dos nossos produtos.

Pelo melhor entendimento da amostragem nós tivemos um excelente retorno com esse projeto uma vez que, com fatos e dados técnicos, pudemos ajustar os procedimentos de amostragem do nosso consumidor, que apresentava problemas com os resultados de $\mathrm{Fe}$ total, e agora não só o comparativo lote a lote se encontra dentro dos limites determinados pelo variograma, mas também não há mais vício [6] no conjunto de lotes que formam um navio destinado aos clientes no exterior, evitando pagamento de multas e perda de faturamento.

\section{Agradecimentos}

Agradecemos a ArcelorMittal Mineração Brasil pela oportunidade e confiança no trabalho desenvolvido, ao gerente Elton Carlos de Souza pelo apoio e a todos os colegas do laboratório e beneficiamento que acreditaram e colaboraram para que tivessemos sucesso no desenvolvimento do trabalho. 


\section{REFERÊNCIAS}

1 M Gy, Pierre M. - Sampling of particulate materials - Theory and practice- Elsevier Scientific Publishing Company - Amsterdam - 1979.

2 Gy, Pierre M. - Sampling of heterogeneous and dynamic material systems - Theory of heterogeneity, sampling and homogenizing - Elsevier Scientific Publishing Company Amsterdam - 1992.

3 ASSOCIAÇÃO BRASILEIRA DE NORMAS TÉCNICAS. ABNT NBR ISO 3082:2011 Minérios de ferro - Procedimento de amostragem e preparação de amostra.

4 ASSOCIAÇÃO BRASILEIRA DE NORMAS TECNICAS. ABNT NBR ISO 3084:2003 Minérios de ferro - Métodos experimentais para avaliação da variação de qualidade. ASSOCIAÇÃO BRASILEIRA DE NORMAS TÉCNICAS. ABNT NBR ISO 3085:2003 Minérios de ferro - Métodos experimentais para verificação da precisão de amostragem, preparação de amostras e medida.

6 ASSOCIAÇÃO BRASILEIRA DE NORMAS TÉCNICAS. ABNT NBR ISO 3086:2008 Minérios de ferro - Métodos experimentais para verificação do vício de amostragem.

7 BARNETT, V. - Sample survey -Principles \& methods - third edition - John Wiley\& Sons -2002.

8 STUART, A. The ideas of sampling. Nova York: MacMillan Publishing Company,1984;;

9 PETERNELLI, L. A.; MELLO, M. P. - Conhecendo o R. Uma visão mais que estatística - Editora Universidade Federal de Viçosa (UFV) - 2013.

10 R DEVELOPMENT CORE TEAM. R: A language and environment for statistical computing.R Foudantion for Statistical Computing, Vienna, Áustria, 2013. Disponível em: www.R-project.org. Acessado em agosto/2013. 